\title{
Capacidad de marcha y dependencia funcional en adultos mayores con alteración visual
}

\section{Gait capacity and functional dependency among older adults with vision disturbances}

\section{Capacidade de marcha e dependência funcional em idosos com alteração visual}

\author{
T. Duran-Badillo ${ }^{\mathrm{a} 1}$, P.L. Hernández-Cortés ${ }^{\mathrm{b} 2}$, M.C. Guevara-Valtier ${ }^{\mathrm{c} 2}$, G. \\ Gutiérrez-Sánchez $^{\mathrm{d} 1}$, M.L. Martínez-Aguilar ${ }^{\mathrm{e} 1}$, M.E. Salazar-Barajas ${ }^{\mathrm{f1}{ }^{*}}$ \\ ORCID: \\ ${ }^{\mathrm{a}} 0000-0002-7274-3511 \quad{ }^{\mathrm{b}} 0000-0001-9185-9416 \quad{ }^{\mathrm{c}} 0000-0001-7291-3931$ \\ ${ }^{\mathrm{d}} 0000-0002-5919-3914 \quad{ }^{\mathrm{e}} 0000-0001-9003-907 X \quad{ }^{\mathrm{f}} 0000-0001-6230-2547$ \\ ${ }^{1}$ Unidad Académica Multidisciplinaria Matamoros, Universidad Autónoma de Tamaulipas, Tamaulipas, México \\ ${ }^{2}$ Facultad de Enfermería, Universidad Autónoma de Nuevo León, Nuevo León, México
}

Recibido: 20 enero 2019

Aceptado: 30 mayo 2019

\section{Resumen}

Introducción: La alteración visual puede producir cambios en la marcha y afectar la autonomía del adulto mayor, entendida como la necesidad de ayuda de otras personas o aditamentos para realizar, adecuadamente, sus actividades de la vida diaria.

Objetivo: Conocer la relación entre la capacidad de marcha y dependencia funcional en adultos mayores con alteración de la agudeza visual.

Material y métodos: Estudio descriptivo y correlacional, en una población de adultos mayores con alteración de la agudeza visual, muestra conformada por 94 adultos mayores. La marcha se midió con el sistema GAITRite, para la dependencia en Actividades Básicas de la Vida Diaria, se utilizó el Índice de Barthel, para las Actividades Instrumentales de la Vida Diaria el índice de Lawton y Brody.

Resultados: La capacidad de marcha y dependencia funcional, presentó relación positiva entre la velocidad de marcha $(p=0.000)$ y longitud del paso $(p=0.000)$ con las ABVD; la velocidad de marcha $(p=0.000)$, cadencia $(p=0.023)$ y longitud del paso $(p=0.000)$ con las AIVD y relación negativa entre la amplitud del paso $(p=0.012)$ y AIVD. 
Conclusión: La valoración de la marcha en los AM con alteración en la agudeza visual, desde el primer nivel de atención, permitirá desarrollar intervenciones y programas encaminados a preservar la independencia del adulto mayor, reducir las tasas de morbilidad, disminuir costos en salud y contribuir en una mejor calidad de vida.

Palabras clave: Adulto mayor; agudeza visual; marcha; actividades de la vida diaria; México.

\section{Abstract}

Introduction: Disturbances in the visual function of the elderly can lead to gait changes which can have an impact on their autonomy, leading to the need of appliances and help of other persons in order for them to adequately perform their daily activities.

Objective: To gain understanding on the relationship between gait capacity and functional dependence among older adults with vision disturbances.

Materials and Methods: This is a descriptive and correlational study on a sample of 94 older adults with vision disturbances. The gait was measured using the GAITRite System. The functional status in terms of Activities of Daily Living (ADL), and Instrumental Activities of Daily Living (IADL) were estimated using the Barthel, and the Lawton \& Brody scales respectively.

Results: The gait capacity and functional dependence showed a positive relationship in terms of velocity $(p=0.000)$ and steps $(p=0.000)$ regarding the ADL; and in terms of velocity $(p=0.000)$, cadence $(p=0.023)$, and steps $(p=0.000)$ regarding the IADL. A negative relationship between step amplitude $(p=0.012)$ and IADL was also found. Conclusion: From the primary healthcare point of view, accurate gait assessments on the older adults with vision disturbances will allow the development of programs and interventions aimed at preserving the independence, reducing the morbidity rates and costs, and contributing to maintain a better quality of life among older adults.

Keywords: Aged; visual acuity; gait; activities of daily life; Mexico.

\section{Resumo}

Introdução: A alteração visual pode produzir câmbios na marcha e afetar a autonomia do idoso, entendida como a necessidade de ajuda de outras pessoas ou aditamentos para realizar, adequadamente, suas atividades da vida diária.

Objetivo: Conhecer a relação entre a capacidade de marcha e dependência funcional em idosos com alteração da agudeza visual.

Material e métodos: Estudo descritivo e correlacional, em uma população de idosos com alteração da agudeza visual, amostra conformada por 94 idosos. A marcha mediu-se com o sistema GAITRite, para dependência em Atividades Básicas da Vida Diária, utilizou-se o Índice de Barthel, para as Atividades Instrumentais da Vida Diária o índice de Lawton e Brody.

Resultados: A capacidade de marcha e dependência funcional, apresentou relação positiva entre a velocidade de marcha $(p=0.000)$ e longitude de passo $(p=0.000)$ com as ABVD; a velocidade de marcha $(p=0.000)$, cadência $(p=0.023)$ e longitude do passo $(p=0.000)$ com as AIVD e relação negativa entre a amplitude do passo $(p=0.012)$ e AIVD.

Conclusão: A valoração da marcha nos AM com alteração na agudeza visual, desde o primeiro nível de atenção, permitirá desenvolver intervenções e programas encaminhados a preservar a independência do idoso, reduzir as taxas de morbilidade, diminuir custos em saude e contribuir em uma melhor qualidade de vida.

Palavras chave: Idoso; agudeza visual; marcha; atividades da vida diária; México.

\section{Introducción}

El envejecimiento poblacional es un fenómeno que ocurre a nivel mundial. La Organización Mundial de la Salud (OMS), plantea que 29 de los países del mundo están en envejecimiento, entre estos se 
encuentra México, con una edad promedio de 76.7 años ${ }^{1}$. El Consejo Nacional de Población indica que en México, en el 2015 la población de adultos mayores era del 9.6\%, con respecto a la población total, aunque en este momento se trata de un país joven, se estima que para el 2030 estas cifras aumenten al $14.9 \%$, para el 2050, la pirámide poblacional se invierta y la población de adultos mayores se incremente hasta alcanzar aproximadamente el 24.7 \%. Al comparar la transición demográfica de los estados de México, en el 2030 las entidades más envejecidas se encontrarán en la zona Norte, por lo que Tamaulipas, será uno de los estados más envejecidos del país².

Vivir más años se puede considerar un importante logro de la humanidad, no obstante, la vejez conlleva cambios físicos, entre ellos, los relacionados con la agudeza visual, la marcha y la dependencia.

La vista es uno de los sentidos de gran importancia, para conocer el contexto en que la persona se encuentra y realizar sus actividades de la vida diaria. Es decir, contribuye a que el individuo conozca el entorno que lo rodea, pueda trasladarse sin dificultad y realizar sus actividades de la vida diaria con independencia ${ }^{3}$. La pérdida de la agudeza visual no es única, existe diversidad, entre ellas, destaca la degeneración macular relativa a la edad, conocida como la causa más común de ceguera legal, con prevalencia proporcional al aumento de la edad ${ }^{4}$, catarata, glaucoma, retinopatía diabética y defectos refractivos no corregidos, causantes de incrementar las tasas de morbimortalidad en la mayoría de los países y causa principal de secuelas e invalidez ${ }^{5}$.

La Organización Mundial de la Salud indica que más del $80 \%$ de las personas con discapacidad visual tienen más de 50 años $^{6}$, dato que resulta preocupante, debido a que la literatura indica que la alteración de la agudeza visual está relacionada con la marcha y la dependencia. La marcha es el conjunto de movimientos rítmicos del tronco y extremidades que determinan un desplazamiento hacia adelante, la alteración en la misma abarca la longitud del paso, amplitud del paso, cadencia y velocidad de marcha ${ }^{7}$.

Los cambios en la marcha afectan la autonomía del adulto mayor, pueden ser el origen de accidentes, caídas, fracturas y causa de muerte. Los adultos mayores como resultado del envejecimiento es más probable que presenten alteraciones del equilibrio, estabilidad y fuerza muscular ${ }^{8}$. El surgimiento de la dependencia desde la perspectiva de los adultos mayores, es entendida como la necesidad de ayuda de otras personas o utilización de aditamentos para realizar, adecuadamente, sus actividades de la vida diaria?

En México la dependencia funcional constituye un serio problema de salud pública, se ha reportado que más del $40 \%$ de los adultos mayores son dependientes para realizar una o más de las Actividades de la Vida Diaria ${ }^{10}$. Las alteraciones en la marcha y la dependencia funcional son problemas que incrementan la vulnerabilidad de los adultos mayores con alteración de la agudeza visual; ante esta problemática, la implicación para los profesionales de enfermería es identificar los factores relacionados para desarrollar intervenciones, ya que de no intervenir de manera adecuada y oportuna, el riesgo de caídas aumenta y con ello la probabilidad de presentar problemas de salud, lo que incrementa los años de vida saludables perdidos por discapacidad, como las fracturas de cadera y el sedentarismo, lo que conlleva a la dependencia para realizar las Actividades Básicas de la Vida Diaria (ABVD), y Actividades Instrumentales de laVida Diaria (AIVD)

En un estudio realizado en México por Hernández et al, reportaron que los problemas de visión incrementan el riesgo de dependencia funcional ${ }^{11}$. Por otra parte, Almeida et al., encontraron que la velocidad de marcha lenta es un factor que incrementa el riesgo de dificultad para realizar una o más actividades de la vida diaria ${ }^{12}$.

En México y otros países en desarrollo, los adultos mayores son un grupo vulnerable que requiere ser atendido desde el primer nivel de atención. En este sentido el Gobierno de la República en el Plan Nacional de Desarrollo 2013-2018 incluyó políticas y programas para la protección de los derechos de las personas con discapacidad ${ }^{13}$. No obstante, la población con algún tipo de discapacidad como la alteración en la agudeza visual sigue siendo vulnerable. Por lo que se considera que los resultados del estudio pueden ser útiles para establecer la relación entre los fenómenos estudiados y contribuir en la prevención de la dependencia funcional en adultos mayores. El objetivo del presente estudio fue determinar la 
relación entre la capacidad de marcha y dependencia funcional en adultos mayores con alteración de la agudeza visual.

\section{Materiales y métodos}

Se trató de un estudio descriptivo y correlacional, en una población de adultos mayores de Monterrey, Nuevo León, México, que son atendidos en un Centro de Salud, La muestra fue conformada por 94 adultos mayores con alteración de la agudeza visual, identificados a través de un muestreo por conveniencia, se tomó como criterio la alteración de la agudeza visual, la cual fue medida con la Carta Snellen de letras o dibujos para los adultos mayores, que no saben leer y escribir, se consideró alteración cuando el adulto mayor resultó con parámetros superiores a 20/20, por lo menos en uno de los ojos, aún con uso de anteojos. En la Tabla 1, se presentan los resultados de la evaluación de agudeza visual de los participantes.

Tabla 1. Agudeza visual de los adultos mayores

\begin{tabular}{ccccc}
\hline \multirow{2}{*}{ Agudeza Visual } & \multicolumn{2}{c}{ Ojo Izquierdo } & \multicolumn{2}{c}{ Ojo Derecho } \\
\cline { 2 - 5 } & $\mathbf{F}$ & \% & $\mathbf{f}$ & $\%$ \\
\hline $20 / 100$ & 37 & 39.4 & 34 & 36.2 \\
\hline $20 / 70$ & 15 & 16.0 & 17 & 18.1 \\
\hline $20 / 50$ & 20 & 21.3 & 14 & 14.9 \\
\hline $20 / 40$ & 22 & 23.4 & 29 & 30.9 \\
\hline
\end{tabular}

Participantes. Los criterios de inclusión fueron: adultos mayores con alteración de la agudeza visual atendidos en un Centro de Salud de primer nivel de atención perteneciente a la Secretaría de Salud, como criterios de exclusión se consideraron edad menor a 60 años y sin alteración de la agudeza visual.

La capacidad de marcha fue medida con el sistema de GAITRite (Tapete de Marcha McaCIRSystems/ GAITRite® Modelo 20'; New Jersey, USA), consiste en un tapete electrónico de $90 \mathrm{~cm}$ de ancho y 550 cm de largo, se conectó a una computadora de la marca HP, modelo RTL8188EE, provista de un software (Standard GAITRite) en el cual se procesaron las pisadas de los adultos mayores. Los parámetros de la marcha que se analizaron fueron: velocidad de marcha $(\mathrm{cm} / \mathrm{s})$, cadencia (pasos/min), longitud del paso $(\mathrm{cm})$ y amplitud del paso $(\mathrm{cm})$. Este sistema reporta una confiabilidad para su medición de 0.91 a $0.99^{14}$.

Para medir la dependencia se utilizó el Índice de Barthel. Su objetivo es evaluar las capacidades funcionales del anciano, se considera su autonomía física e instrumental en las ABVD de mantenimiento: comer, lavarse, vestirse, arreglarse, usar el retrete (control de esfínteres, deposiciones, micción), subir/bajar escaleras, trasladarse (o manejar una silla de ruedas) y deambular. Los resultados globales se agruparon en cuatro categorías de dependencia: 1) menos de 21 puntos se calificó como dependencia total, 2) de 21 a 60 puntos dependencia severa, 3) de 61 a 90 puntos dependencia moderada, 4) de 91 a 99 puntos dependencia escasa y, 5) 100 puntos total independencia. El alpha de Cronbach reportado para este instrumento es de 0.86 a $0.92^{15}$. Las AIVD se midieron con el Índice de Lawton y Brody. Las actividades que incluye esta escala son la capacidad de los AM para usar el teléfono, hacer compras, preparar comidas, cuidar el hogar, lavar la ropa, usar los medios de transporte, asumir la responsabilidad respecto de la medicación y el manejo de asuntos económicos. Se consideró independiente cuando reportó realizar las ocho actividades que evalúa el instrumento, con una fiabilidad interclase de $0.94^{16}$.

El estudio se apegó a los principios éticos para la investigación médica con seres humanos, plasmados en la Declaración de Helsinki de la Asociación Médica Mundial ${ }^{17}$. De manera inicial, se solicitó la aprobación de las autoridades correspondientes para realizar el trabajo de campo (DEISC-19 0116 01), se acudió al centro de salud, se hizo una invitación abierta a los adultos mayores para realizarse un examen de la vista, se entregaron los resultados y a los que cumplieron con los criterios de inclusión, se les hizo la invitación a participar en el estudio, se les explicó todo lo relacionado, se hizo lectura del consentimiento 
informado y se solicitó que lo firmaran, previa aclaración que podían retirarse en el momento que así lo decidieran, sin repercusión en la atención que habitualmente reciben en la institución, finalmente, se hicieron las mediciones y se cuidó en todo momento la integridad de las personas.

Los datos fueron procesados y analizados en el programa estadístico Statistical Package for the Social Sciences (SPSS), versión 20 para Windows. Inicialmente, se verificó la normalidad con la prueba de Kolmogorov Smirnov con corrección de Lilliefors y en función de los resultados, se optó por utilizar el coeficiente de correlación Pearson, para responder el objetivo planteado.

\section{Resultados}

En el estudio participaron 94 adultos mayores con alteración en la agudeza visual. El 78.7 \% (74) correspondió a mujeres, el promedio de edad fue de 70.72 años $(\mathrm{DE}=7.06)$ y de escolaridad 8.40 años $(\mathrm{DE}=$ 5.02). El $34.0 \%$ (32) reportó haber sufrido alguna caída en el último año, el 8.5 \% (8) utilizaba bastón o andador para caminar y el 41.5 \% (39) indicó que en el último mes había sufrido mareos.

En cuanto a la dependencia funcional, en las ABVD se observó que el $57.4 \%$ (54) resultó con independencia, el $27.7 \%$ (26) con dependencia escasa, el $13.8 \%$ (13) con dependencia moderada y el $1.1 \%$ (1) con dependencia severa; mientras que en las AIVD el $71.36 \%$ (67) fueron independientes y el $28.7 \%$ (27) con dependencia funcional. En la Tabla 2 se encuentran los datos descriptivos de los parámetros de la marcha y de las ABVD y AIVD.

Tabla 2. Descripción de la capacidad de marcha y dependencia funcional

\begin{tabular}{lrccc}
\hline \multirow{2}{*}{ Variable } & Media & DE & \multicolumn{2}{c}{ IC 95 \% } \\
\cline { 4 - 5 } & & & $\begin{array}{c}\text { Límite } \\
\text { inferior }\end{array}$ & $\begin{array}{c}\text { Límite } \\
\text { superior }\end{array}$ \\
\hline Velocidad de marcha & 91.07 & 21.60 & 86.65 & 95.54 \\
\hline Cadencia & 103.44 & 11.42 & 50.86 & 54.81 \\
\hline Longitud del paso & 52.87 & 9.45 & 50.86 & 54.81 \\
\hline Amplitud del paso & 9.32 & 3.13 & 8.61 & 10.02 \\
\hline ABVD & 95.27 & 8.49 & 93.15 & 96.97 \\
\hline AIVD & 6.99 & 2.06 & 6.52 & 7.40 \\
\hline
\end{tabular}

En el análisis de la relación entre la capacidad de marcha y dependencia funcional, se obtuvo relación positiva y estadísticamente significativa entre la velocidad de marcha $(r=0.382 ; \mathrm{p}=0.000)$ y longitud del paso $(r=00.362 ; p=0.000)$, con las ABVD; así mismo, la velocidad de marcha $(r=0.427 ; p=$ $0.000)$, cadencia $(r=0.234 ; \quad p=0.023)$ y longitud del paso $(r=0.429 ; p=0.000)$, se relacionó positiva y significativamente con las AIVD. En conclusión, se observó relación negativa y estadísticamente significativa entre la amplitud del paso $(\mathrm{r}=-0.258 ; \mathrm{p}=0.012)$ y AIVD (Tabla 3$)$.

Tabla 3. Relación entre parámetros de la marcha y dependencia funcional

\begin{tabular}{lcccc}
\hline \multirow{2}{*}{ Variables } & \multicolumn{2}{c}{ ABVD } & \multicolumn{2}{c}{ AIVD } \\
\cline { 2 - 5 } & $\mathbf{r}$ & $\mathbf{P}$ & $\mathbf{r}$ & $\mathbf{P}$ \\
\hline Velocidad de marcha & 0.382 & 0.000 & 0.427 & 0.000 \\
& & & & \\
\hline Cadencia & 0.183 & 0.077 & 0.234 & 0.023 \\
\hline Longitud del paso & 0.362 & 0.000 & 0.429 & 0.000 \\
\hline Amplitud del paso & -0.071 & 0.498 & -0.258 & 0.012 \\
\hline
\end{tabular}




\section{Discusión}

El envejecimiento conlleva diversos cambios en el funcionamiento de órganos y sistemas. Entre los principales cambios, se encuentra la afección de la agudeza visual que aunado a otros factores ambientales, psicológicos y físicos, contribuye en la alteración de la capacidad de marcha.

Lo anterior se evidencia a través de los hallazgos de este estudio, donde empíricamente se pudo comprobar que la velocidad de marcha resultó por debajo de los parámetros considerados como normales, de acuerdo con Cerda, debe aproximarse a $1 \mathrm{~m} / \mathrm{s}^{15}$. Lo encontrado en relación con la velocidad de marcha es congruente con lo reportado por Almeida et al. La disminución en la velocidad de marcha en adultos mayores con alteración de la agudeza visual, se explica, que al no identificar la distancia en que las cosas se encuentran, como mecanismo de prevención de riesgos, los adultos mayores tienden a caminan más lento ${ }^{12}$.

Este fenómeno, principalmente, pudiera presentarse en sujetos que tuvieron o estuvieron a punto de presentar alguna caída, sin embargo, no es un dato que se haya investigado en el presente, por lo que se sugiere en futuros estudios, profundizar en el efecto de las experiencias previas de caídas sobre la velocidad de la marcha en adultos mayores con alteración en la agudeza visual.

Respecto a la cadencia, se considera normal cuando la persona camina a un ritmo mayor de 90 pa$\mathrm{sos} / \mathrm{min}^{18}$. La media de pasos en este estudio fue superior a los parámetros normales y se aproxima a lo reportado por otros estudios ${ }^{19}$. Pese a que la amplitud y longitud del paso se encontraba dentro de los parámetros normales, la velocidad de marcha fue lenta, situación que sugiere que aunque los adultos mayores dan pasos normales, caminan más lento como un mecanismo de protección, para asegurarse que se encuentran en un espacio firme y sin riesgo.

En el presente estudio, se encontró que a mayor velocidad de marcha y longitud del paso, mayor independencia para realizar las ABVD. A mayor velocidad de marcha, cadencia y longitud del paso, mayor independencia para realizar las AIVD; del mismo modo, se encontró relación negativa entre la amplitud del paso y las AIVD. Estos datos apoyan lo documentado en la literatura, donde se indica que la capacidad de marcha se relaciona con la dependencia para realizar las actividades de la vida diaria ${ }^{3}$. Recientemente, un estudio reportó que los adultos mayores con velocidad de marcha lenta tienen mayor dificultad para realizar las actividades de la vida diaria ${ }^{12}$.

No obstante, es importante mencionar que existen factores que pudieran explicar las alteraciones en la velocidad de marcha, como lo muestra la literatura entre dependencia funcional en adultos mayores con enfermedades oculares (degeneración macular relativa a la edad, glaucoma, retinopatía diabética) ${ }^{4,5}$ y otros más, que sería importante considerar en futuras investigaciones, entre ellos la presencia de problemas osteoarticulares, neuromusculares, ambientales y estado de ánimo.

Por tanto, se justifica la importancia de establecer un diagnóstico temprano de las alteraciones visuales, cambios en la marcha y factores causales, para intervenir oportunamente, debido a que la evidencia científica indica que la afección en la marcha es un factor de riesgo de dependencia funcional ${ }^{20}$. En este estudio, casi la mitad de los adultos mayores presentó algún nivel de dependencia, similar a lo reportado en otro estudio ${ }^{15}$. Un tercio de adultos mayores resultó con dependencia para realizar sus AIVD, éste dato se acerca a lo reportado en otros estudios en los que se midió la dependencia en adultos mayores, que acudieron a la consulta médica o se encontraban en su domicilio ${ }^{7}$.

La relación encontrada entre los parámetros de la marcha y la dependencia funcional, para realizar diversas actividades de la vida diaria se requiere la movilización, por ejemplo, para trasladarse de la cama a la silla, desplazarse, subir y bajar escaleras; este dato es congruente con lo reportado en la literatura, donde se indica que las alteraciones en la marcha del adulto mayor son un predictor de dependencia en ABVD y AIVD, además que puede incrementar el uso de servicios asistenciales al aumentar el riesgo de caídas $^{21}$.

El envejecimiento por sí mismo, conlleva implicaciones políticas, sociales, culturales y económicas ${ }^{21}$. De manera específica, las económicas se incrementan cuando el adulto mayor cursa con trastornos en 
la salud física, como las alteraciones en la marcha, que conllevan a la dependencia, mismas que por las complicaciones pueden ocasionar necesidad de atención especializada o de hospitalización. En un estudio realizado en el Instituto Mexicano del Seguro Social, se encontró que los costos por hospitalización de adultos mayores son elevados y estos son diferentes de acuerdo con el grupo de edad, las personas mayores de 85 años son quienes originan mayor incremento del gasto social ${ }^{22}$. Los hallazgos del presente estudio y los altos costos por hospitalización evidenciados, resaltan la importancia de la atención preventiva y énfasis en la valoración de la marcha y dependencia funcional, en adultos mayores con alteración de la agudeza visual, desde el primer nivel de atención, lo que permitirá reducir los costos en salud y contribuirá en una mejor calidad de vida de los adultos mayores.

\section{Conclusiones}

En este estudio se observó que existe relación entre la velocidad de marcha y longitud del paso con la dependencia para realizar las ABVD, también, la velocidad de marcha, cadencia, longitud y amplitud del paso están relacionados con la dependencia para efectuar las AIVD, en los adultos mayores con alteración de la agudeza visual. Se reconoce que haber utilizado un muestreo no probabilístico por conveniencia, es una limitante para la generalización de los resultados, sin embargo, se considera que los datos obtenidos son un importante acercamiento en el estudio de la marcha y dependencia funcional en esta población vulnerable.

Es de suma importancia, que enfermería al momento de realizar la valoración física en los adultos mayores, considere la capacidad de la marcha y tenga presente la relación que esta tiene con la dependencia en algunas actividades de la vida diaria e instrumentales, con ello contribuir en la prevención de accidentes, caídas y fracturas, que puedan llevar a la dependencia, lo que permitirá reducir los costos en salud y contribuir en una mejor calidad de vida de los adultos mayores.

Por otra parte, resalta la importancia de continuar en esta línea de investigación para generar conocimiento científico que permita al profesional de Enfermería desarrollar e implementar intervenciones. Se recomienda realizar valoración de la marcha y de la dependencia funcional a los adultos mayores que resulten con alteración en la agudeza visual, desde el primer nivel de atención. Así como desarrollar intervenciones y programas con enfoque anticipatorio al riesgo, encaminados a preservar la independencia del adulto mayor, para reducir las tasas de morbilidad que conlleve a que un mayor número de individuos disfruten su vejez en plenitud.

\section{Responsabilidades éticas}

Protección de personas y animales. En esta investigación, no se han realizado experimentos en seres humanos ni animales.

Confidencialidad de los datos. Los autores declaran que en este artículo no aparecen datos de los participantes.

Financiamiento. Los autores declaran no haber recibido financiamiento para realizar esta investigación. Conflicto de intereses. Los autores declaran no tener conflicto de intereses.

\section{Referencias}

1. World Health Organization. World Health Statistics: monitoring health for the SDGs, sustainable development goals. OMS. Geneva; WHO: 2016. https://bit.ly/2WPz0cZ

2. González KD. Envejecimiento demográfico en México: análisis comparativo entre las entidades federativas. En: La situación demográfica de México. CDMX: Consejo Nacional de Población; 2015. https://bit.ly/1Y3Ij0T

3. Eliopoulos C. Enfermería gerontológica. 8ª ed. Barcelona: Wolters Kluwer Health; 2014. 
4. Quintero-Busutil M, Perea Ruíz CA, Padilla-González C M, Rojas-Rondón I, Rodríguez-Masó S, LuisGoytisolo I. Capacidad funcional y calidad de vida en los ancianos con degeneración macular y baja visión. Rev. cuba. oftalmol. 2014; 27 (3): 332-49. https://bit.ly/2wXgMqj

5. Hernández-Narváez MG, Olivares-Luna AM, Carillo-Hernández A, Tovar-Méndez GM, GonzálezPedraza Avilés A. Prevalencia de trastornos visuales y su relación con la funcionalidad en adultos mayores. Rev. cuba. oftalmol. 2015; 28(2): 190-7. https://bit.ly/2WQdzsc

6. Organización Mundial de la Salud. Ceguera y discapacidad visual. Nota descriptiva. Ginebra: OMS; 2018. https://bit.ly/2Tr7bSo

7. Cervantes-Becerra RG, Villarreal-Ríos E, Galicia-Rodríguez L, Vargas-Daza ER, Martínez-González L. Estado de salud en el adulto mayor en atención primaria a partir de una valoración geriátrica integral. Aten. prim. 2015; 47(6): 329-35. https://doi.org/10.1016/j.aprim.2014.07.007

8. Demura T, Demura S, Uchiyama M, Sugiura H. Examination of factors affecting gait properties in healthy older adults: focusing on knee extension strength, visual acuity, and knee joint pain. J Geriatr Phys Ther. 2014; 37 (2): 52-7. http://dx.doi.org/10.1519/JPT.0b013e318295daba

9. Duran-Badillo T, Domínguez-Chávez CJ, Hernández-Cortés PL, Félix-Alemán A, Cruz-Quevedo JE, Alonso-Castillo MM. Dejar de ser o hacer: significado de dependencia funcional para el adulto mayor. Acta Universitaria. 2018; 28(3): 40-6. https://doi.org/10.15174/au.2018.1614

10. Organización Mundial de la Salud. Informe mundial sobre el envejecimiento y la salud. Ginebra: OMS: 2015. https://bit.ly/2FiS08W

11. Hernández-Baguer R, Triana-Casado I, Bueno-Arrieta Y, Cid-Vázquez B. Capacidad funcional, repercusión psicológica y social y calidad de vida de ancianos con degeneración macular involutiva. Rev haban cienc méd. 2013; 12(3): 387-98. https://bit.ly/2XjMjBN

12. Busch T de A, Duarte YA, Pires-Nunes D, Lebrão ML, Satya-Naslavsky M, Dos Santos-Rodrigues A, et al. Factors associated with lower gait speed among the elderly living in a developing country: a crosssectional population-based study. BMC Geriatr. 2015; 15(35): 1-9.

http://dx.doi.org/10.1186/s12877-015-0031-2

13. Gobierno de la República. Plan Nacional de Desarrollo 2013- 2018. Programa Sectorial de Salud. CDMX; Secretaría de Salud: 2013. https://bit.ly/23Sdy1n

14. Webster KE, Wittwer JE, Feller JA. Validity of the GAITRite walkway system for the measurement of averaged and individual step parameters of gait. Gait Posture. 2004; 22(4): 317-21.

https://doi.org/10.1016/j.gaitpost.2004.10.005

15. Cid-Ruzafa J, Damián-Moreno J. Valoración de la discapacidad física: el índice de Barthel. Rev. esp. salud publica. 1997; 71(2): 127-137. https://bit.ly/2AdJ6XS

16. Trigás-Ferrín M, Ferreira-González L, Meijide-Míguez H. Escalas de valoración funcional en el anciano. Galicia Clín. 2011; 72(1): 11-6. https://bit.ly/2qN7N8o

17. Asociación Médica Mundial. Declaración de Helsinki deAMM-Principios éticos para las investigaciones médicas en seres humanos. Adoptada por la $18^{a}$ Asamblea Médica Mundial, Helsinki, Finlandia, junio 1964 y enmendada por la 52 Asamblea General, Edimburgo, Escocia, octubre 2000. Escocia: AMM; 2000. https://bit.ly/2r2W2cs

18. Cerda L. Evaluación del paciente con trastorno de la marcha Rev Hosp Clín Univ Chile. 2010; (21): 226-36. https://bit.ly/2J2dXdx

19. Jerome GJ, Ko SU, Kauffman D, Studenski SA, Ferrucci L, Simonsick EM. Gait characteristics associated with walking speed decline in older adults: Results from the Baltimore longitudinal study of aging. Arch Gerontol Geriatr. 2015; 60(2): 239-43. http://dx.doi.org/10.1016/j.archger.2015.01.007

20. Runzer-Colmenares FM, Castro G, Merino A, Torres-Mallma C, Diaz G, Perez C, Asociación entre depresión y dependencia funcional en pacientes adultos mayores. Horiz. Med. 2017; 17(3): 50-7. http://dx.doi.org/10.24265/horizmed.2017.v17n3.09 
21. Sánchez-García S. Envejecimiento y Salud. Rev. Med. Inst. Mex. Seguro Soc. $2018 ; 56$ (Supl 1): S4-5. https://bit.ly/2Knmmeq

22. Granados-García V, Sánchez-García S, Ramírez-Aldana R, Zúñiga-Trejo C, Espinel-Bermúdez MC. Costos por hospitalización de adultos mayores en un hospital general regional del IMSS. Rev. Med. Inst. Mex. Seguro Soc. 2018; 56(Supl 1): S64-70. https://bit.ly/2MVfKGf 